\title{
XPD inhibits cell growth and invasion and enhances chemosensitivity in esophageal squamous cell carcinoma by regulating the PI3K/AKT signaling pathway
}

\author{
JIE JIAN $^{1 *}$, SHUANG LI $^{2 *}$, LI-ZHEN LIU ${ }^{3}$, LI ZHEN $^{1}$, LING YAO $^{1}$, \\ LI-HONG GAN ${ }^{1}$, YA-QING HUANG ${ }^{1}$ and NIAN FANG ${ }^{1}$
}

\begin{abstract}
Departments of ${ }^{1}$ Gastroenterology and ${ }^{2}$ Geriatrics and General Medicine, Third Affiliated Hospital of Nanchang University, Nanchang, Jiangxi 330008; ${ }^{3}$ Department of Oncology, Jiading District Central Hospital Affiliated

Shanghai University of Medicine and Health Sciences, Shanghai 201800, P.R. China
\end{abstract}

Received January 20, 2020; Accepted April 13, 2020

DOI: $10.3892 /$ ijmm.2020.4593

\begin{abstract}
Esophageal squamous cell carcinoma (ESCC) is a lethal disease due to its high aggressiveness. The aim of the present study was to investigate the role of xeroderma pigmentosum complementation group D (XPD) in the growth and invasion of ESCC and to elucidate the potential underlying molecular mechanisms. Western blot analysis and RT-qPCR were used to detect the expression level of XPD in ESCC tissue samples and adjacent normal esophageal tissue samples. The pEGFP-N2/XPD plasmid was transfected into human ESCC cell lines (EC9706 and EC109). The proliferation, apoptosis, migration and invasion of EC9706 or EC109 cells were assessed following transfection with the XPD overexpression plasmid. The chemosensitivity of EC9706 or EC109 cells to cisplatin or fluorouracil was evaluated by CCK-8 assay. The expression levels of phosphoinositide 3-kinase (PI3K)/AKT, nuclear factor (NF)- $\kappa \mathrm{B}$, Janus kinase 2 (JAK2)/signal transducer and activator of transcription 3 (STAT3) and mitogen-activated protein kinase (MAPK) signaling pathway-related genes were detected by RT-qPCR and western blot analysis. The results demonstrated that the expression level of XPD was markedly lower in ESCC tissue samples than in adjacent normal esophageal tissue samples. The pEGFP-N2/XPD plasmid was successfully transfected into EC9706 or EC109 cells, inducing XPD overexpression. A High XPD expression markedly suppressed cell proliferation, migration and invasion,
\end{abstract}

Correspondence to: Dr Nian Fang, Department of Gastroenterology, Third Affiliated Hospital of Nanchang University, 128 Xiangshan North Road, Nanchang, Jiangxi 330008, P.R. China

E-mail: fnian3893@126.com

*Contributed equally

Key words: xeroderma pigmentosum complementation group D, esophageal squamous cell carcinoma, proliferation, apoptosis, PI3K/AKT signaling pathway and increased the apoptotic rate of EC9706 and EC109 cells. Furthermore, the overexpression of XPD significantly increased the chemosensitivity of EC9706 and EC109 cells to cisplatin or fluorouracil. Following XPD overexpression, the expression levels of PI3K, p-AKT, c-Myc, Cyclin D1, Bcl-2, vascular endothelial growth factor (VEGF) and matrix metalloproteinase (MMP)-9 were markedly downregulated, while the expression level of p21 was markedly upregulated. On the whole, the findings of the present study demonstrate that XPD inhibits the growth and invasion of EC9706 and EC109 cells, whilst also enhancing the chemosensitivity of EC9706 and EC109 cells to cisplatin or fluorouracil by regulating the PI3K/AKT signaling pathway. XPD may thus be an underlying target for ESCC treatment and drug resistance.

\section{Introduction}

Esophageal squamous cell carcinoma (ESCC), a major histological type of esophageal cancer, is one of the most lethal malignant cancers. In 2018, there were an estimated 455,800 new cases and 400,200 deaths related to ESCC worldwide (1). The 5-year survival rate of patients with ESCC is $<10 \%$ in China, and $<20 \%$ in the United States. In China, ESCC is the main type of esophageal cancer, and its incidence has gradually increased over the past decade (2). ESCC can be caused by a variety of factors, including heavy smoking, alcohol consumption, foods rich in nitrosamines or contaminated with mycotoxins, Barrett's esophagus and esophageal reflux disease (3). Traditional treatments for ESCC include surgical resection, chemotherapy and radiotherapy. However, the majority of patients still suffer from tumor recurrence and metastasis following complete ESCC resection (4). Chemotherapy is a widely used alternative in ESCC treatment; however, drug resistance is widespread (5). Therefore, it is imperative to explore the pathogenesis of ESCC and develop novel therapeutic targets for ESCC treatment and drug resistance.

Malignant tumors result from the instability of the cell genome, which can occur when processes including DNA repair, DNA replication and chromosome segregation are 
altered. Genetic mutation is the most common cause of DNA damage. Xeroderma pigmentosum complementation group D (XPD) can regulate the transcription initiation and cleavage repair of damaged nucleotide sequences and maintain the biological process in a normal and orderly fashion (6). XPD plays an important role in the repair of damage caused by oxidative stress (7). A previous study found that XPD gene polymorphism increases the risk of lung cancer in residents of coal mines (8). XPD polymorphisms are associated with the development of pre oral cancer as well as oral cancer and its clinical course (9). It has been found that XPD can inhibit the proliferation and migration of hepatocellular carcinoma cells (10). However, the biological roles of XPD in ESCC remain unclear.

In the present study, the mechanisms through which XPD participates in the tumorigenesis and progression of ESCC were investigated. An XPD gene-encoding plasmid was transfected into ESCC cell lines (EC9706 and EC109 cells), and changes in the molecular biological behavior of EC9706 or EC109 cells were observed. In addition, the molecular mechanisms underlying the XPD-mediated regulation of ESCC cell growth and invasion were investigated.

\section{Materials and methods}

Reagents. Dulbecco's modified Eagle's medium (DMEM) and fetal bovine serum (FBS) were purchased from Gibco; Thermo Fisher Scientific, Inc. The vacant vector plasmid pEGFP-N2 and the recombinant plasmid pEGFP-N2/XPD were generously provided by Jiangxi Provincial Key Laboratory of Molecular Medicine and these plasmids have been described in previous studies $(10,11)$. Lipofectamine $^{\mathrm{TM}} 2000$ and TRIzol reagent were purchased from Invitrogen; Thermo Fisher Scientific, Inc. PCR primers were synthesized by Sangon Biotech. The total protein extraction kit was purchased from AmyJet Scientific Inc. The reverse transcription kit was purchased from Fermentas; Thermo Fisher Scientific, Inc. The Annexin V-FITC/PI kit was purchased from Vazyme Biotech. The Cell Counting kit-8 was purchased from Solarbio Science Technology. Transwell chambers were purchased from BD Biosciences. Anti-XPD (ab54676) primary antibody was purchased from Abcam. Primary antibodies against phosphoinositide 3-kinase (PI3K; \#4249), AKT (\#4685), p-AKT (Ser473) (\#4060), Bcl-2 (\#15071), p21 (\#2947), p-p65 (Ser536) (\#3033), p65 (\#8242), p-signal transducer and activator of transcription 3 (STAT3; Tyr705) (\#9145), STAT3 (\#12640), p-p38 mitogen-activated protein kinase (MAPK; Thr180/Tyr182) (\#4511), p38 MAPK (\#8690) and $\beta$-actin (\#4970) were purchased from Cell Signaling Technology, Inc. Horseradish peroxidase-conjugated secondary antibodies (ZB-2305 and ZB-2306) were purchased from Beijing Zhongshan Golden Bridge Biotechnology Co. Ltd. Cisplatin, fluorouracil and LY294002 were purchased from MedChemExpress (MCE).

Clinical specimens. A total of 20 ESCC tissue samples and adjacent normal esophageal tissue samples ( $>5 \mathrm{~cm}$ away from the tumor) were collected from patients who underwent gastroscopy, endoscopic biopsy and pathological diagnosis at the Department of Gastroenterology of Third Affiliated
Hospital of Nanchang University (Nanchang, China) between September, 2018 and December, 2018. The 20 ESCC cases were obtained from 14 males and 6 females aged 41-77 years. No patient had received radiotherapy or chemotherapy prior to the endoscopic biopsy. The present study was approved by the Human Ethics Committee of Third Affiliated Hospital of Nanchang University and prior written consent was obtained from all patients.

Cells and cell culture. The human ESCC cell lines, EC9706 and EC109, were obtained from the American Type Culture Collection (ATCC). All cells were cultured in DMEM supplemented with $10 \%$ FBS, $100 \mathrm{U} / \mathrm{ml}$ penicillin and $100 \mu \mathrm{g} / \mathrm{ml}$ streptomycin and incubated in a humidified incubator at $37^{\circ} \mathrm{C}$, $5 \% \mathrm{CO}_{2}$ and $95 \%$ air.

Cell transfection. The EC9706 or EC109 Cells were divided into 3 groups as follows: i) The untransfected control group (Ctrl); ii) pEGFP-N2 empty plasmid transfection group (pEGFP-N2); and iii) the pEGFP-N2/XPD plasmid transfection group (pEGFP-N2/XPD). The detailed procedures for transfection were described in a previous study by the authors (12). At $48 \mathrm{~h}$ following the transfection of the pEGFP-N2 or pEGFP-N2/XPD plasmids, green fluorescence was observed under a fluorescence microscope (Olympus Corp.). Western blot analysis and RT-qPCR were used to detect the protein and mRNA expression levels of XPD.

Cell proliferation assay. At $0,24,48,72$ or $96 \mathrm{~h}$ following transfection with XPD plasmid, the EC9706 or EC109 cells were seeded into 96 -well culture plates at a density of $4 \times 10^{3}$ cells/well. In addition, for the pEGFP-N2/XPD + LY294002 group, the EC9706 or EC109 cells were treated with $10 \mu \mathrm{mol} / 1$ of LY294002 for 0, 24, 48, 72 or $96 \mathrm{~h}$ following transfection with XPD plasmid. The cells in each group were washed with PBS and incubated with $100 \mu \mathrm{l} \mathrm{CCK}-8$ solution for $1 \mathrm{~h}$ at $37^{\circ} \mathrm{C}$. The absorbance at $450 \mathrm{~nm}$ was measured using a microplate reader (Thermo Fisher Scientific, Inc.). Each independent experiment was performed 3 times. Data were calculated as the means $\pm \mathrm{SD}$.

Cell apoptosis assay. The EC9706 or EC109 cells in each group were trypsinized and collected by centrifugation at $37^{\circ} \mathrm{C}$ for $5 \mathrm{~min}$ at a speed of $1,000 \mathrm{x} \mathrm{g}$. A total of $1 \times 10^{5}$ cells were then resuspended in $500 \mu \mathrm{l}$ of buffer and incubated with Annexin V-FITC/PI kit for $15 \mathrm{~min}$ at room temperature. The apoptotic rate of EC9706 or EC109 cells was detected using a flow cytometer (FACSCalibur, BD Biosciences). Each experiment was performed in triplicate independently.

Cell Transwell migration and invasion assays. Cell migration and invasion assays were performed using Transwell chambers without or with Matrigel according to the manufacturer's instructions and as previously described (12). A total of $2 \times 10^{5}$ EC9706 or EC109 cells were seeded into the upper chamber of the insert in serum-free DMEM. The lower chamber of the insert contained DMEM supplemented with 10\% FBS as a chemoattractant. Following incubation in a humidified incubator at $37^{\circ} \mathrm{C} 5 \% \mathrm{CO}_{2}$ and $95 \%$ air for $48 \mathrm{~h}, \mathrm{EC} 9706$ or EC109 cells remaining on the insert's top layer were wiped 
off with a cotton swab. Cells that migrated or invaded to the lower surface of the membrane were stained with crystal violet (Beijing Solarbio Science \& Technology Co., Ltd.) for $20 \mathrm{~min}$ at room temperature and imaged under an inverted light microscope (x50 magnification). Cells in 5 fields were counted to calculate the cell migration or invasion. Each experiment was performed in triplicate.

Chemosensitivity assay. At $24 \mathrm{~h}$ following transfection with the XPD plasmid, EC9706 or EC109 cells were seeded into 96-well plates at a density of $4 \times 10^{3}$ cells/well. The medium was then discarded and the cells were incubated at $37^{\circ} \mathrm{C}$ in the presence of $0,20,40,80$ and $100 \mu \mathrm{g} / \mathrm{ml}$ cisplatin or fluorouracil for 72 h. EC9706 or EC109 cell sensitivity to cisplatin or fluorouracil in each group was detected by CCK-8 assay as described above. Cell viability (\%) was calculated by using the following formula: OD value $(0,20,40,80$, or $100 \mu \mathrm{g} / \mathrm{ml}) / \mathrm{OD}$ value $(0 \mu \mathrm{g} / \mathrm{ml}) \times 100 \%$.

$R T-q P C R$. Total RNA was isolated from all tissue samples, EC9706, or EC109 cells according to the standard TRIzol method. A total of $1 \mu \mathrm{g}$ RNA was used as a template for cDNA synthesis using a reverse transcription kit at $37^{\circ} \mathrm{C}$ for $15 \mathrm{~min}$ and $85^{\circ} \mathrm{C}$ for $30 \mathrm{sec}$. XPD, PI3K, AKT, Bcl-2, c-Myc, p21, Cyclin D1, vascular endothelial growth factor (VEGF), matrix metalloproteinase (MMP)-9 and $\beta$-actin primers were designed using Primer Premier 5.0 software. The primer sequences are presented in Table I. Quantitative PCR was performed using TB Green Premix Ex Taq (Takara Bio, Inc.) under the following thermal cycling conditions: Initial denaturation at $95^{\circ} \mathrm{C}$ for $5 \mathrm{~min}$; subsequent 40 cycles of $95^{\circ} \mathrm{C}$ for $30 \mathrm{sec}$ and $60^{\circ} \mathrm{C}$ for $30 \mathrm{sec}$. The relative expression mRNA levels of XPD, PI3K, AKT, Bcl-2, c-Myc, p21, Cyclin D1, VEGF and MMP-9 were calculated using the $2^{-\Delta \Delta C q}(13)$ method. $\beta$-actin was used as an endogenous control. All reactions were repeated 3 times.

Western blot analysis. Total protein of all tissue samples, EC9706 or EC109 cells was extracted using a total protein extraction kit. The protein concentration was measured using the BCA assay kit according to the manufacturer's instructions. Protein samples were separated by $10 \%$ SDS polyacrylamide gel electrophoresis and transferred to nitrocellulose membranes. The membranes were blocked with $5 \%$ non-fat milk in Tris buffered saline containing Tween-20. The membranes were then incubated with specific primary antibodies against XPD (1:1,000), PI3K $(1: 1,000)$, AKT $(1: 1,000)$, p-AKT (1:2,000), Bcl-2 (1:1,000), p21 (1:2,000), p65 (1:1,000), p-p65 (1:1,000), STAT3 (1:1,000), p-STAT3 (1:2,000), p38 MAPK $(1: 1,000)$, p-p38 MAPK $(1: 1,000)$ and $\beta$-actin $(1: 3,000)$ at $4^{\circ} \mathrm{C}$ overnight, followed by incubation with horseradish peroxidase-conjugated secondary antibodies (1:800) for $1 \mathrm{~h}$ at room temperature. Finally, the signal was visualized using ECL reagent. ImageJ 1.8.0 software was used to determine the density of the protein bands. Anti- $\beta$-actin antibody was used as an endogenous control.

Statistical analysis. All experiments were performed at least 3 times and all final data were expressed as the mean \pm SD Graphpad prism 7.0 software was used to evaluate the experimental data through one-way analysis of variance (ANOVA)
Table I. Primer sequences used for RT-qPCR.

\begin{tabular}{ll}
\hline Gene & \multicolumn{1}{c}{ Primer sequences } \\
\hline XPD & F: 5'-TCTGCCTCTGCCCTATGAT-3' \\
& R: 5'-CGATTCCCTCGGACACTTT-3' \\
PI3K & F:5'-TGGCCTTAGCTCTTAGCCAAACAC-3' \\
& R: 5'-ATTGGAACACGGCCTTTGACA-3' \\
AKT & F: 5'-CTGTGCCTATGCTGCCCAT-3' \\
& R: 5'-CAGTGCGATGTCGTGGAGG-3' \\
Bcl-2 & F: 5'-GGATAACGGAGGCTGGGATGC-3' \\
& R: 5'-GACTTCACTTGTGGCCCAGAT-3' \\
c-Myc & F: 5'-TGTGTTACGGTCGCGTCTTT-3' \\
& R: 5'-AACAGCTCGGTCACCATCTC-3' \\
p21 & F: 5'-GACCTGTCACTGTCTTGTAC-3' \\
& R: 5'-CTCTCATTCAACCGCCTAG-3' \\
Cyclin D1 & F: 5'-CCAGACCCACGTTTCTTTGC-3' \\
& R: 5'-ATCCCTAGAAACACCACGGC-3' \\
VEGF & F: 5'-ACCCACTCACTGGCTGTTT-3' \\
& R: 5'-CGGGCTCTGAGATGTTCAG-3' \\
MMP-9 & F: 5'- TAGACGCTGCTCCCCTCA-3' \\
& R: 5'-GCGGGTGTAACCATAGCG-3' \\
F-actin & F: 5'-AAGGTGACAGCAGTCGGTT-3' \\
& R: 5'-TGTGTGGACTTGGGAGAGG-3' \\
&
\end{tabular}

XPD, xeroderma pigmentosum complementation group D; PI3K, phosphoinositide 3-kinase; VEGF, vascular endothelial growth factor; MMP-9, matrix metalloproteinase 9, F, forward, R, reverse.

among groups and the LSD post hoc test. The t-test was used to determine differences between 2 groups. Differences in the expression levels of XPD in ESCC tissue samples were evaluated using a Fisher's exact test or Pearson's $\chi^{2}$ test. Differences at $\mathrm{P}<0.05$ were considered statistically significant.

\section{Results}

$X P D$ expression is downregulated in human ESCC tissue samples. To examine the expression level of XPD in ESCC, 20 pairs of human ESCC tissue samples and adjacent normal esophageal tissue samples were collected to detect the mRNA and protein expression levels of XPD. The mRNA and protein expression levels of XPD were markedly decreased in human ESCC tissue samples compared to adjacent normal esophageal tissue samples (Figs. 1 and S1). The association between XPD expression and various clinicopathological characteristics is shown in Table II. XPD expression was associated with histological grade $(\mathrm{P}=0.011)$ and clinical $\mathrm{TNM}$ stage $(\mathrm{P}=0.032)$, indicating that XPD may be associated with the progression of ESCC.

Successful transfection of XPD plasmid and upregulation of $X P D$. The pEGFP-N2/XPD plasmid or pEGFP-N2 plasmid were transiently transfected into EC9706 cells and EC109 cells. At $48 \mathrm{~h}$ following transfection, the cells were observed under a fluorescence microscope. As shown in Fig. 2A, cells successfully transfected with pEGFP-N2/XPD or pEGFP-N2 
A

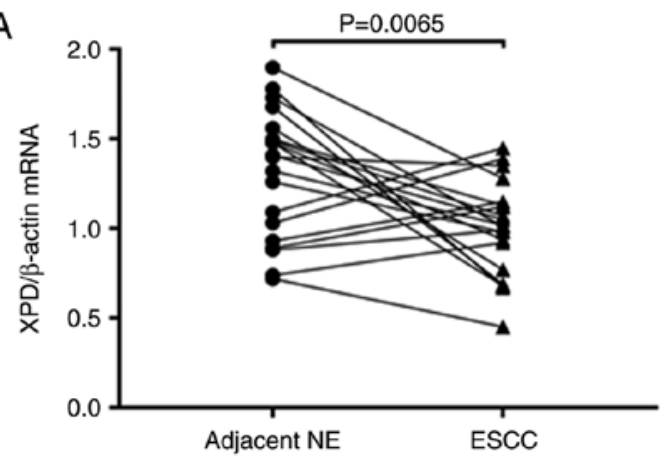

B

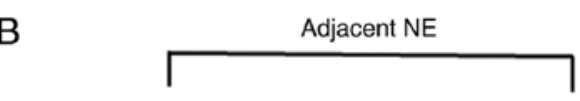

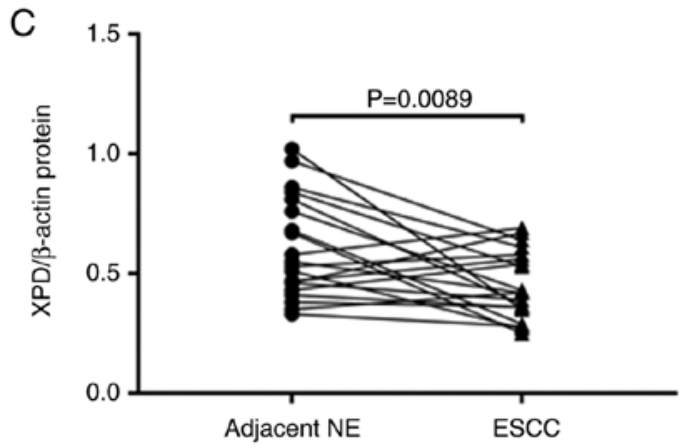

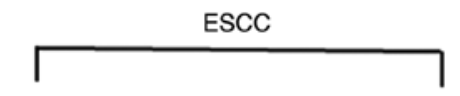

$\begin{array}{lllll}\text { T1 } & \text { T2 } & \text { T3 } & \text { T4 } & \text { T5 }\end{array}$

XPD
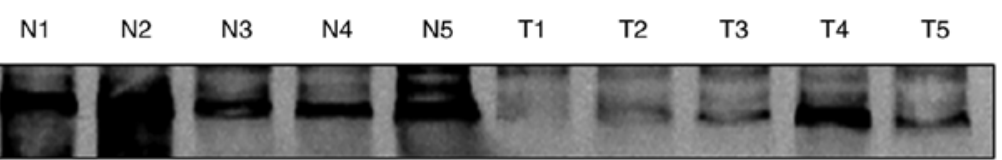

$\beta$-actin

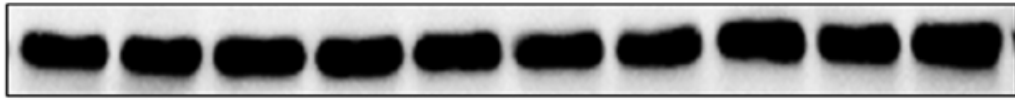

Figure 1. XPD expression in ESCC and adjacent normal esophageal tissues. The expression levels of XPD (A) mRNA and (B and C) protein in ESCC tissue samples and adjacent normal esophageal tissue samples were detected by RT-qPCR and western blot analysis, respectively. Data represent the means \pm standard deviation. ESCC, esophageal squamous cell carcinoma; XPD, xeroderma pigmentosum complementation group D; Adjacent NE, adjacent normal esophagus.

Table II. Association between XPD expression and clinicopathological factors of ESCC patients.

\begin{tabular}{lrrrrr}
\hline & \multicolumn{4}{c}{$\begin{array}{c}\text { XPD } \\
\text { expression }\end{array}$} \\
Factors & $\begin{array}{c}\text { No. of } \\
\text { patients }\end{array}$ & Low & High & P-value \\
\hline Sex & & & & 0.354 \\
$\quad$ Male & 14 & 8 & 6 & \\
Female & 6 & 5 & 1 & \\
Age (years) & & & & 0.174 \\
$\quad<55$ & 7 & 3 & 4 & \\
$\geq 55$ & 13 & 10 & 3 & \\
Histological (differentiation) & & & & 0.011 \\
grade & & & & \\
Well & 1 & 0 & 1 & \\
Moderately & 5 & 1 & 4 & \\
Poorly & 14 & 12 & 2 & \\
TNM stage & & & & 0.032 \\
I & 2 & 0 & 2 & \\
II & 7 & 3 & 4 & \\
III & 6 & 5 & 1 & \\
IV & 5 & 5 & 0 & \\
\hline & & & &
\end{tabular}

plasmid exhibited green fluorescence. No green fluorescence was observed in the control (Ctrl) group. The results of western blot analysis and RT-qPCR revealed that the mRNA (Fig. 2B and C) and protein (Fig. 2D-F) expression levels of
XPD in EC9706 cells or EC109 cells of the pEGFP-N2/XPD group were markedly upregulated.

XPD overexpression suppresses the proliferation of EC9706 cells and EC109 cells. Cell viability was used to investigate whether XPD overexpression affects the proliferation of EC9706 or EC109 cells. At 24, 48, 72 or 96 h following transfection with XPD plasmid, the optical density (OD) value of the pEGFP-N2/XPD group was significantly reduced compared to the that of the control (Ctrl) group and pEGFP-N2 group $(\mathrm{P}<0.05$ or $\mathrm{P}<0.01$; Fig. $3 \mathrm{~A}$ and $\mathrm{B})$. There was no significant difference in the OD value between the $\mathrm{Ctrl}$ group and pEGFP-N2 group (P>0.05; Fig. 3A and B). XPD overexpression can thus inhibit the proliferation of EC9706 and EC109 cells.

Upregulation of XPD increases the apoptosis of EC9706 and EC109 cells. As shown in Fig. 3C-E, compared with the Ctrl group and the pEGFP-N2 group, the apoptotic rate of the EC9706 or EC109 cells in the pEGFP-N2/XPD group was significantly higher $(\mathrm{P}<0.001)$. There was no marked difference in the cell apoptotic rate between the Ctrl group and the pEGFP-N2 group ( $P>0.05)$. XPD upregulation can thus promote the apoptosis of EC9706 and EC109 cells.

$X P D$ overexpression inhibits the migration and invasion of EC9706 and EC109 cells. Transwell migration and invasion assays were used to examine the effects of XPD overexpression on the migration and invasion of EC9706 or EC109 cells. As shown in Fig. 3F-H, compared to the Ctrl group and pEGFP-N2 group, the migratory capacity of the EC9706 or EC109 cells in the pEGFP-N2/XPD group was markedly decreased $(\mathrm{P}<0.01)$. Compared to the Ctrl group and the pEGFP-N2 group, the 
A

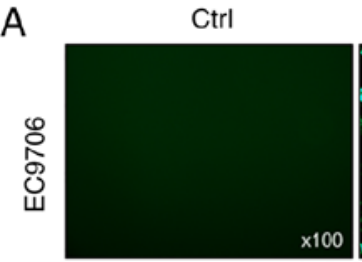

은

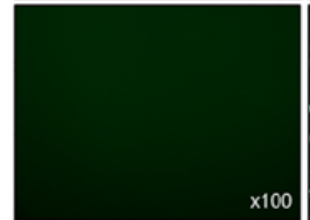

B

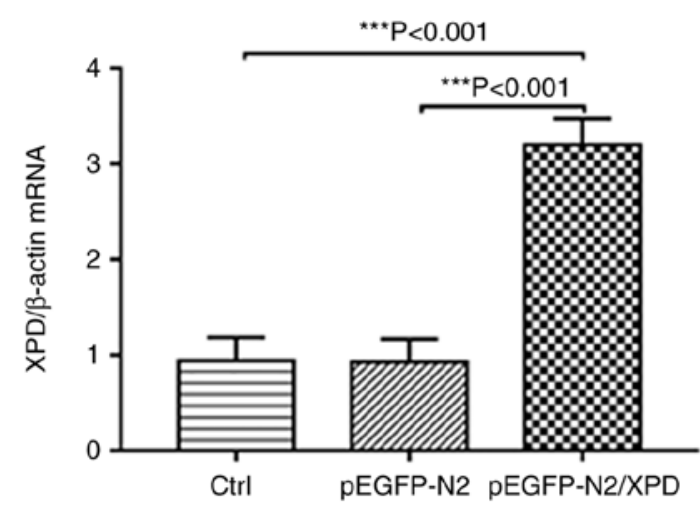

E

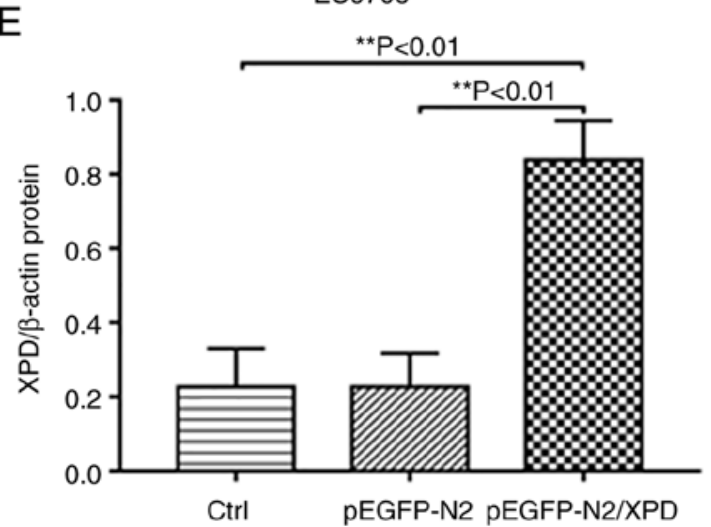

PEGFP-N2/XPD
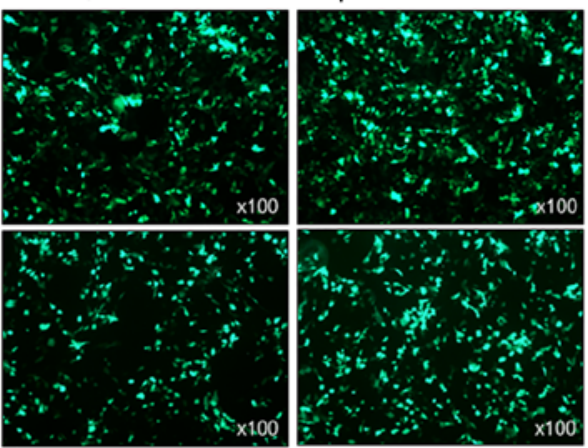

D

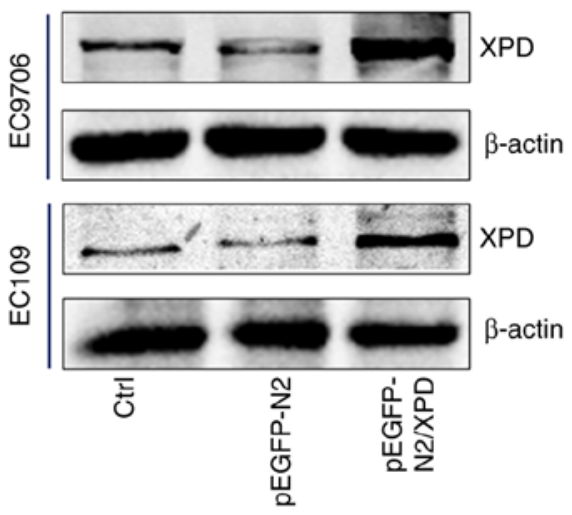

C

EC109

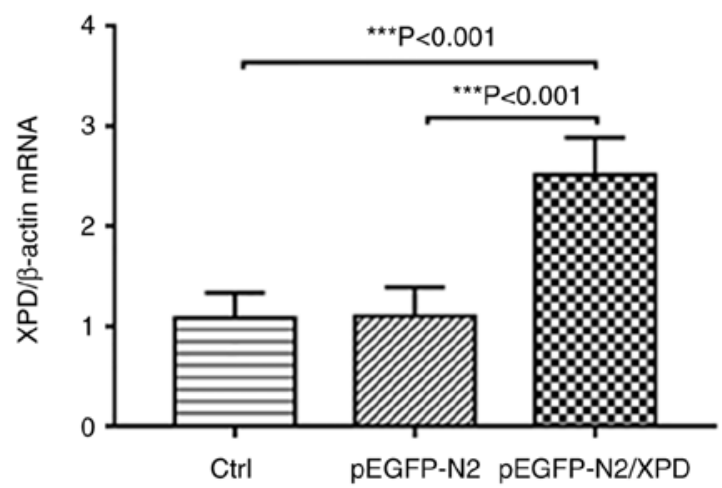

$\mathrm{F}$

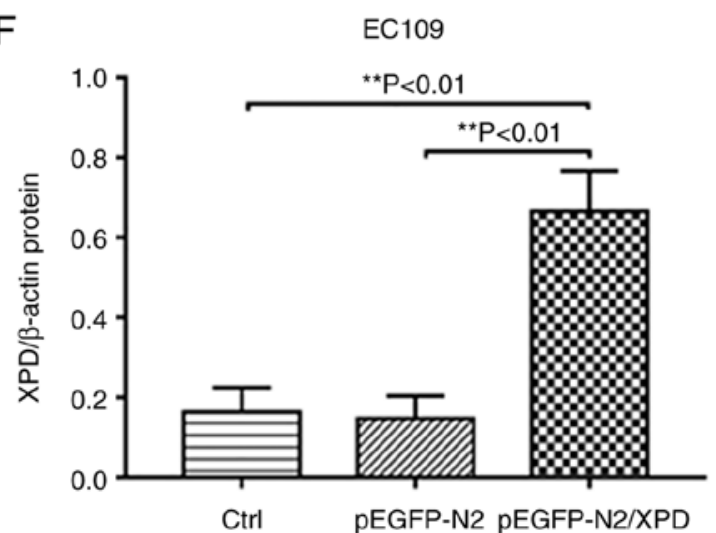

Figure 2. XPD expression is upregulated following transfection with pEGFP-N2/XPD plasmid. (A) Green fluorescent protein was observed after pEGFP-N2 or pEGFP-N2/XPD plasmid transfection (x100 magnification). The expression levels of XPD (B and C) mRNA and (D-F) protein in EC9706 or EC109 cells were detected by RT-qPCR and western blot analysis, respectively. ${ }^{* *} \mathrm{P}<0.01$ or ${ }^{* * * *} \mathrm{P}<0.001$, vs. Ctrl group or pEGFP-N2 group (n=3). pEGFP-N2/XPD, pEGFP-N2/XPD plasmid was transfected into EC9706 or EC109 cells; pEGFP-N2, pEGFP-N2 plasmid was transfected into EC9706 or EC109 cells; Ctrl, no transfection; XPD, xeroderma pigmentosum complementation group D.

upregulation of XPD in the EC9706 cells or EC109 cells significantly reduced the invasive ability $(\mathrm{P}<0.001$; Fig. 3I-K). There was no significant difference in the migratory and invasive capacities between the pEGFP-N2 group and Ctrl group (P>0.05; Fig. 3F-K). XPD upregulation can thus inhibit the migration and invasion of EC9706 or EC109 cells.

Overexpression of XPD enhances the chemosensitivity of EC9706 and EC109 cells to cisplatin or fluorouracil. At $24 \mathrm{~h}$ following transfection with XPD plasmid, the EC9706 or EC109 cells were incubated in the presence of $0,20,40,80$ and $100 \mu \mathrm{g} / \mathrm{ml}$ cisplatin or fluorouracil for $72 \mathrm{~h}$. As shown in Fig. 4, the results of CCK- 8 assay revealed that compared with the Ctrl group and the pEGFP-N2 group, the chemosensitivity of the EC9706 and EC109 cells to cisplatin or fluorouracil in the pEGFP-N2/XPD group was markedly enhanced $(\mathrm{P}<0.05$ or $\mathrm{P}<0.01)$. XPD overexpression can thus increase the chemosensitivity of EC9706 and EC109 cells to cisplatin or fluorouracil.

Upregulation of XPD inhibits the PI3K/AKT signaling pathway. Previous studies have found that the tumorigenesis and progression of ESCC are associated with the PI3K/AKT signaling pathway $(14,15)$. In the present study, RT-qPCR and 

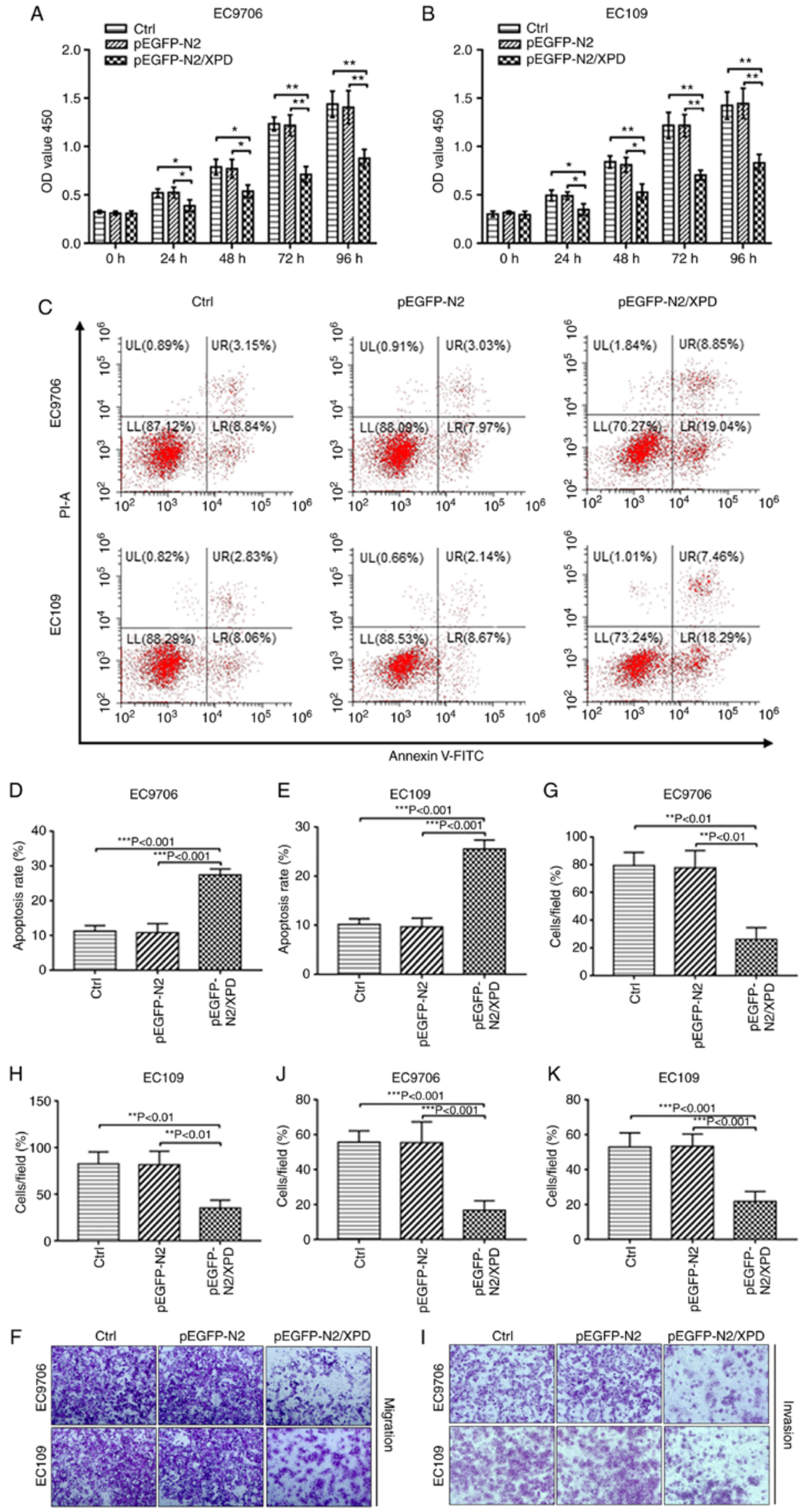

Figure 3. Effects of XPD overexpression on the proliferation, apoptosis, migration and invasion of EC9706 and EC109 cells. (A and B) CCK-8 assay was used to detect the proliferation of EC9706 or EC109 cells. (C-E) Flow cytometry was used to detect the apoptosis of EC9706 or EC109 cells. The effects of XPD overexpression on the migration (F-H) and the invasion (I-K) of EC9706 or EC109 cells were examined by Transwell assay. Data represent the means \pm standard deviation. ${ }^{*} \mathrm{P}<0.05,{ }^{* *} \mathrm{P}<0.01$ or ${ }^{* * *} \mathrm{P}<0.001$, vs. Ctrl group or pEGFP-N2 group (n=3). pEGFP-N2/XPD, pEGFP-N2/XPD plasmid was transfected into EC9706 or EC109 cells; pEGFP-N2, pEGFP-N2 plasmid was transfected into EC9706 or EC109 cells; Ctrl, no transfection; XPD, xeroderma pigmentosum complementation group D. 

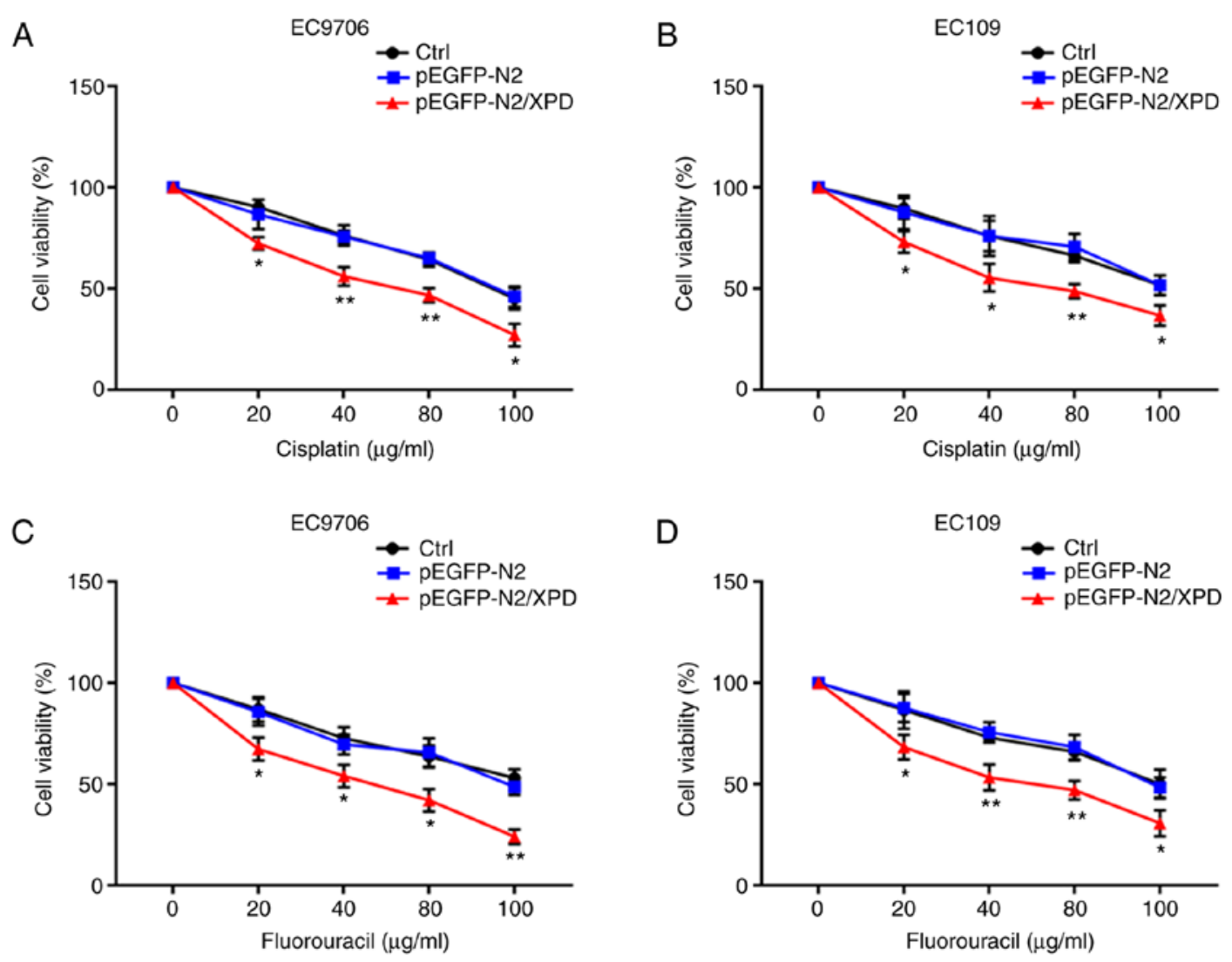

Figure 4. Upregulation of XPD enhances the chemosensitivity of EC9706 and EC109 cells to cisplatin or fluorouracil. The effects of XPD upregulation on the chemosensitivity of EC9706 and EC109 cells to (A and B) cisplatin or (C and D) fluorouracil were determined by CCK-8 assay. Date represent the means \pm standard deviation. ${ }^{*} \mathrm{P}<0.05$ or ${ }^{* *} \mathrm{P}<0.01$, vs. Ctrl group or pEGFP-N2 group ( $\mathrm{n}=3$ ). pEGFP-N2/XPD, pEGFP-N2/XPD plasmid was transfected into EC9706 or EC109 cells; pEGFP-N2, pEGFP-N2 plasmid was transfected into EC9706 or EC109 cells; Ctrl, no transfection; XPD, xeroderma pigmentosum complementation group D.

western blot analysis were used to investigate the potential effects of XPD overexpression on the expression levels of PI3K/AKT signaling pathway-related factors in the EC9706 or EC109 cells. As shown in Fig. 5A and B, compared with the pEGFP-N2 group and the Ctrl group, the mRNA expression levels of PI3K, Bcl-2, c-Myc, Cyclin D1, VEGF and MMP-9 in the EC9706 or EC109 cells of pEGFP-N2/XPD group were markedly decreased $(\mathrm{P}<0.05$ or $\mathrm{P}<0.01)$, whereas the mRNA expression of p21 was markedly increased $(\mathrm{P}<0.01)$. There was no significant difference in mRNA expression level of AKT among the pEGFP-N2/XPD, pEGFP-N2 and Ctrl groups $(\mathrm{P}>0.05)$. As shown in Fig. 5C-F, compared to the pEGFP-N2 group and the $\mathrm{Ctrl}$ group, the protein expression levels of $\mathrm{PI} 3 \mathrm{~K}$, $\mathrm{P}-\mathrm{AKT}$ and $\mathrm{Bcl}-2$ in the pEGFP-N2/XPD group were markedly decreased $(\mathrm{P}<0.05$ or $\mathrm{P}<0.01)$, whereas the protein expression level of p21 in the pEGFP-N2/XPD group was significantly upregulated $(\mathrm{P}<0.05$ or $\mathrm{P}<0.01)$. There was no significant difference in the protein expression level of AKT among the pEGFP-N2/XPD, pEGFP-N2 and Ctrl groups $(\mathrm{P}>0.05)$. In addition, there was no significant difference in the expression levels of PI3K/AKT signaling pathway-associated factors between the pEGFP-N2 group and the Ctrl group ( $\mathrm{P}>0.05)$. To further investigate the involvement of the PI3K/AKT pathway in XPD-inhibited ESCC progression, the PI3K/AKT pathway inhibitor, LY294002 $(10 \mu \mathrm{M})$ was used. Following transfection with XPD plasmid, the EC9706 or EC109 cells in the pEGFP-N2/XPD + LY294002 group were treated with
$10 \mu \mathrm{mol} / 1$ of LY294002 for $0,24,48,72$ or $96 \mathrm{~h}$. The proliferative abilities of the EC9706 or EC109 cells were then detected by CCK- 8 assay. The results revealed that XPD overexpression markedly inhibited the proliferative capabilities of the EC9706 or EC109 cells, and LY294002 significantly enhanced the suppressive effects (Fig. $5 \mathrm{G}$ and $\mathrm{H}, \mathrm{P}<0.05$ or $\mathrm{P}<0.01$ ). To determine the association between XPD and other signaling pathways in ESCC, such as the NF- $\mathrm{B}$, JAK2/STAT3 and MAPK signaling pathways (16-18), the protein expression levels of p65, p-p65, STAT3, p-STAT3, p38 MAPK and p-p38 MAPK were detected in the EC9706 cells following the overexpression of XPD. As shown in Fig. 5I and J, there was no significant difference in the protein expression levels of NF- $\kappa$ B p65, JAK2/STAT3, and p38 MAPK signaling pathways among the pEGFP-N2/XPD, pEGFP-N2 and Ctrl groups $(\mathrm{P}>0.05)$. These results indicated that the upregulation of XPD can inhibit the PI3K/AKT signaling pathway in ESCC.

\section{Discussion}

ESCC is one of the most common malignancies and the most commonly diagnosed type of esophageal cancer (19). ESCC ranks as the 6th most lethal cancer worldwide due to its high mortality $(19,20)$. Esophageal cancer is often diagnosed at an advanced stage and the patient survival rate is very low due to the lack of specific symptoms and effective early diagnostic methods (21). Although multiple treatments, such 
A

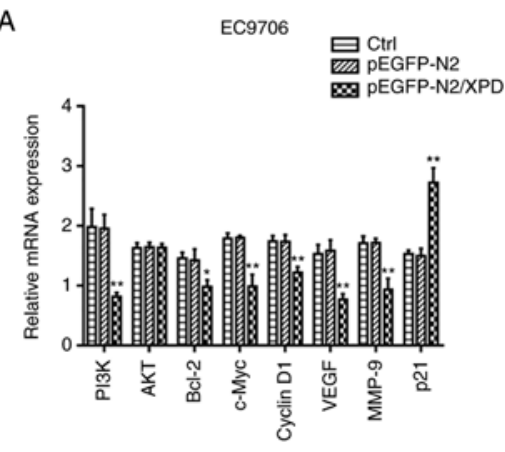

C

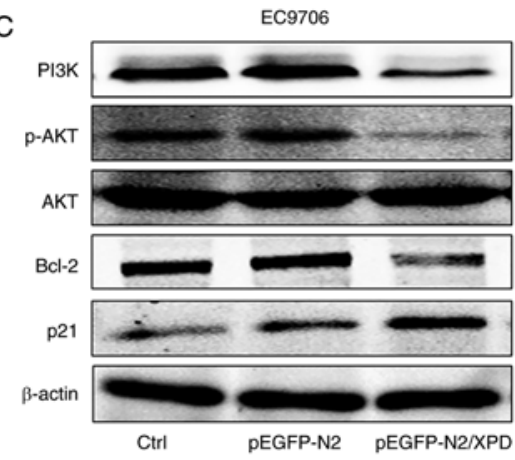

$E$
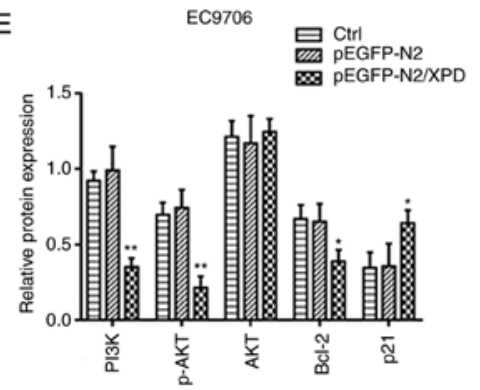

G
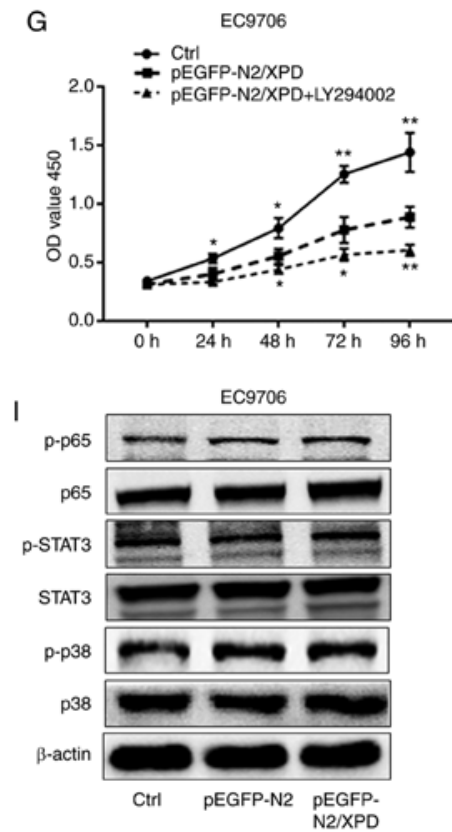

$J$
B
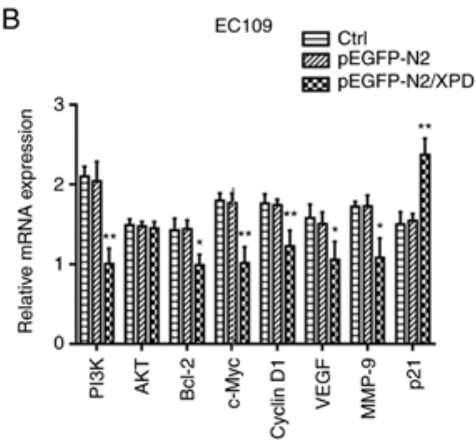

D

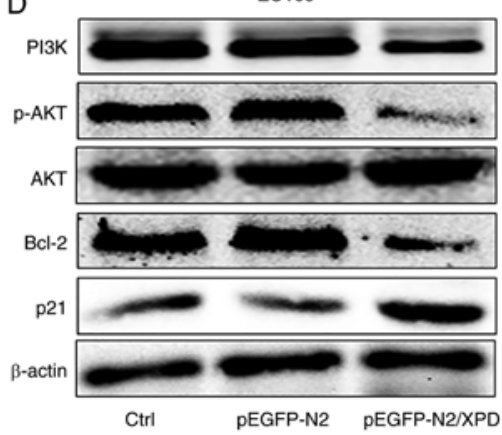

F
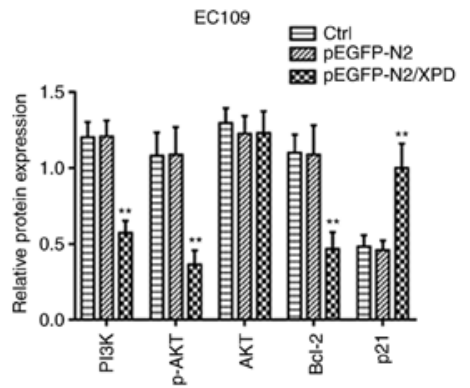

H
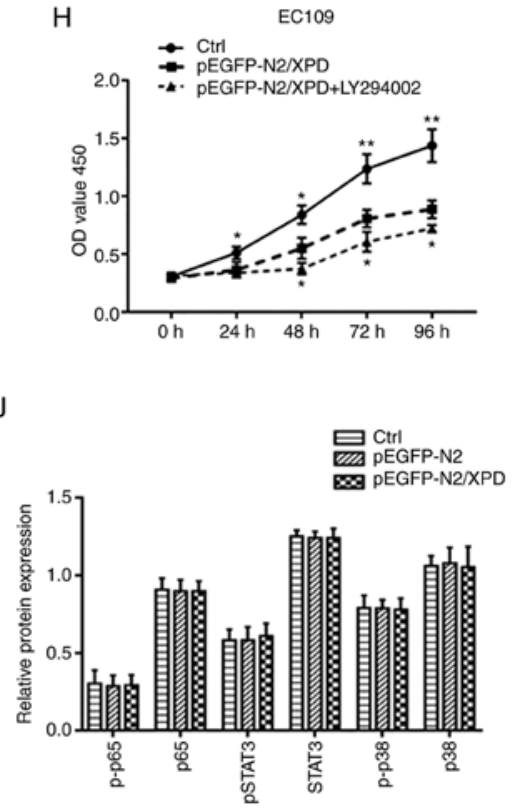

Figure 5. Effects of XPD overexpression on multiple signaling pathways in EC9706 cells and EC109 cells. (A and B) The mRNA expression levels of PI3K, AKT, Bcl-2, c-Myc, Cyclin D1, VEGF, MMP-9 and p21 in EC9706 or EC109 cells were detected by RT-qPCR. "P<0.05 or ${ }^{* *} \mathrm{P}<0.01$, vs. Ctrl group or pEGFP-N2 group ( $\mathrm{n}=3)$. (C-F) The protein expression levels of PI3K, p-AKT, AKT, Bcl-2 and p21 in EC9706 or EC109 cells were detected by western blot analysis. (G and H) Following treatment with or without LY294002 $(10 \mu \mathrm{M})$, cell proliferation was examined by CCK-8 assay. ${ }^{*} \mathrm{P}<0.05$ or ${ }^{* * *} \mathrm{P}<0.01$, vs. pEGFP-N2/XPD group (n=3). (I and J) The protein expression levels of p-p65, p65, p-STAT3, STAT3, p-p38, and p38 in EC9706 cells were detected by western blot analysis. Data represent the means \pm standard deviation. pEGFP-N2/XPD, pEGFP-N2/XPD plasmid was transfected into EC9706 or EC109 cells; pEGFP-N2, pEGFP-N2 plasmid was transfected into EC9706 or EC109 cells; Ctrl, no transfection; pEGFP-N2/XPD + LY294002, pEGFP-N2/XPD plasmid was transfected into EC9706 or EC109 cells and cells were then treated with LY294002 (10 $\mu \mathrm{M})$; XPD, xeroderma pigmentosum complementation group D. 
as surgical resection, chemotherapy, and radiotherapy have been applied for ESCC treatment, the prognosis of ESCC remains poor (22-24). Therefore, it is of utmost importance to better understand the molecular mechanisms responsible for the development of ESCC and to explore novel therapies with which to improve the survival of patients with ESCC.

XPD is located on 19q13.2-q13.3 and encodes an ATP-dependent DNA helicase (25). XPD751 polymorphism has been shown to be associated with the occurrence and development of a wide range of malignancies, such as esophageal cancer, gastric cancer, and colorectal cancer (26-28). Previous studies have demonstrated that XPD expression serves as a tumor suppressor in HCC $(10,11,29)$. In a present study by the authors, it was found that the mRNA and protein expression levels of XPD in ESCC tissue samples were significantly lower than those in the adjacent normal esophageal tissue samples. To determine the role of XPD in ESCC, the in vitro cellular effects of XPD overexpression in ESCC were investigated through XPD transfection into EC9706 and EC109 cells. In the present study, it was demonstrated that XPD gene overexpression significantly reduced the proliferation and inhibited the migration and invasion of EC9706 or EC109 cells, whilst increasing cell apoptosis. Additionally, the upregulation of XPD gene enhanced the chemosensitivity of EC9706 and EC109 cells to cisplatin or fluorouracil.

Previous studies have indicated that the PI3K/AKT signaling pathway plays an important role in the occurrence, development and invasion of malignant tumors, such as esophageal cancer, colon cancer and gastric cancer (30-32). A previous study by the authors demonstrated that the inhibition of the activation of AKT and the promotion of the expression of p21 inhibited cell proliferation and promotes cell apoptosis in hepatocellular carcinoma (12). In the present study, XPD was shown to be involved in the phosphorylation of AKT. Following XPD upregulation, the protein expression level of $\mathrm{p}$-AKT was significantly decreased, indicating that XPD overexpression may inhibit the activation of AKT and suppress PI3K/AKT signal transduction. $\mathrm{p} 21$ has been demonstrated to be involved in cell cycle progression and apoptosis, as well as in behaviors essential for tumorigenesis and tumor progression (33). The present study also demonstrated that the mRNA and protein expression levels of p21 were significantly upregulated following the overexpression of XPD. Previous studies have demonstrated that c-Myc, Cyclin D1 and Bcl-2 play crucial roles in regulating tumorigenesis and are significantly upregulated during tumor progression (34-36). The present study also revealed that the expression levels of c-Myc, Cyclin D1 and Bcl-2 were significantly downregulated following the overexpression of XPD. Previous studies have demonstrated that the functions of VEGF and MMP-9 are essential for tumor invasion $(37,38)$. The present study also demonstrated that the mRNA expression levels of VEGF and MMP-9 were both significantly decreased following the overexpression of XPD. As shown by the results of the present study, the expression level of XPD was low in ESCC tissues, EC9706 cells, or EC109 cells; thus, XPD overexpression experiments were conducted and this is the reason that XPD knockdown experiments were not conducted in the EC9706 or EC109 cells. As such, the fact that there no XPD knockdown experiments were performed is a limitation of the present study.
In conclusion, the findings of the present study demonstrate that the upregulation of XPD inhibits the proliferation, abrogates the migration and invasion, and promotes the apoptosis of EC9706 and EC109 cells by inhibiting the PI3K/AKT signaling pathway. XPD overexpression also enhanced the chemosensitivity of EC9706 and EC109 cells to cisplatin or fluorouracil. Based on the results of the present study, XPD may thus become a potential target for ESCC treatment and drug resistance in the future.

\section{Acknowledgements}

The authors would like to thank Dr Bin Li, Dr Jian-Bin Qin and Dr Feng Deng (Department of Gastroenterology, Third Affiliated Hospital of Nanchang University) for providing their valuable assistance with this research.

\section{Funding}

The present study was supported by the National Natural Science Foundation of China (grant. no. 81660408) and the Health and Family Planning Commission Science and Technology Plan of Jiangxi Province (grant. no. 20184002).

\section{Availability of data and materials}

The datasets used in the present study can be obtained from the corresponding author upon reasonable request.

\section{Authors' contributions}

JJ, SL and NF designed the experiments. JJ, SL, LZL, LZ, LY, LHG and YQH performed the experiments. SL contributed to the data analysis. JJ wrote the manuscript and conducted the revision of the manuscript. NF was responsible for the final modification of the manuscript. All authors read and approved the final manuscript.

\section{Ethics approval and consent to participate}

The present study was approved by the Human Ethics Committee of Third Affiliated Hospital of Nanchang University and prior written consent was obtained from all patients.

\section{Patient consent for publication}

Not applicable.

\section{Competing interests}

The authors declare that they have no competing interests.

\section{References}

1. Bray F, Ferlay J, Soerjomataram I, Siegel RL, Torre LA and Jemal A: Global cancer statistics 2018: GLOBOCAN estimates of incidence and mortality worldwide for 36 cancers in 185 countries. CA Cancer J Clin 68: 394-424, 2018.

2. Yutong H, Xiaoli X, Shumei L, Shan S, Di L and Baoen S: Increased neutrophil-lymphocyte ratio is a poor prognostic factor in patients with esophageal cancer in a high incidence area in China. Arch Med Res 46: 557-563, 2015. 
3. Toh Y, Oki E, Ohgaki K, Sakamoto Y, Ito S, Egashira A, Saeki H, Kakeji Y, Morita M, Sakaguchi Y, et al: Alcohol drinking, cigarette smoking, and the development of squamous cell carcinoma of the esophagus: Molecular mechanisms of carcinogenesis. Int J Clin Oncol 15: 135-144, 2010.

4. Moon DH, Jeon JH, Yang HC, Kim YI, Lee JY, Kim MS, Lee JM and Lee GK: Intramural metastasis as a risk factor for recurrence in esophageal squamous cell carcinoma. Ann Thorac Surg 106: 249-256, 2018

5. Wu H, Chen S, Yu J, Li Y, Zhang XY, Yang L, Zhang H, Hou Q, Jiang M, Brunicardi FC, et al: Single-cell transcriptome analyses reveal molecular signals to intrinsic and acquired paclitaxel resistance in esophageal squamous cancer cells. Cancer Lett 420 : $156-167,2018$

6. Oksenych V and Coin F: The long unwinding road: XPB and XPD helicases in damaged DNA opening. Cell Cycle 9: 90-96, 2010.

7. Lerner LK, Moreno NC, Rocha CRR, Munford V, Santos V, Soltys DT, Garcia CCM, Sarasin A and Menck CFM $\mathrm{XPD} / \mathrm{ERCC} 2$ mutations interfere in cellular responses to oxidative stress. Mutagenesis 34: 341-354, 2019.

8. Minina VI, Bakanova ML, Soboleva OA, Ryzhkova AV, Titov RA, Savchenko YA, Sinitsky MY, Voronina EN, Titov VA and Glushkov AN: Polymorphisms in DNA repair genes in lung cancer patients living in a coal-mining region. Eur J Cancer Prev 28: 522-528, 2019.

9. Nigam K, Yadav SK, Samadi FM, Bhatt ML, Gupta S and Sanyal S: Risk modulation of oral pre cancer and cancer with polymorphisms in XPD and XPG genes in North Indian population. Asian Pac J Cancer Prev 20: 2397-2403, 2019.

10. Xiao Z, Wang Y and Ding H: XPD suppresses cell proliferation and migration via miR-29a-3p-Mdm2/PDGF-B axis in HCC. Cell Biosci 9: 6, 2019.

11. Ding H, Xu JJ, Huang Y, Du FT and Zhang JX: XPD could suppress growth of HepG2.2.15 and down-regulate the expression of hepatitis B virus x protein through P53 pathway. Biochem Biophys Res Commun 419: 761-767, 2012.

12. Huang DH, Jian J, Li S, Zhang Y and Liu LZ: TPX2 silencing exerts anti-tumor effects on hepatocellular carcinoma by regulating the PI3K/AKT signaling pathway. Int J Mol Med 44: 2113-2122, 2019.

13. Livak KJ and Schmittgen TD: Analysis of relative gene expression data using real-time quantitative PCR and the 2(-Delta Delta C(T)) method. Methods 25: 402-408, 2001.

14. Wang L, Zhang Z, Yu X, Li Q, Wang Q, Chang A, Huang X, Han X, Song Y, Hu J, et al: SOX9/miR-203a axis drives $\mathrm{PI} 3 \mathrm{~K} / \mathrm{AKT}$ signaling to promote esophageal cancer progression. Cancer Lett 468: 14-26, 2020.

15. Zheng TL, Li DP, He ZF and Zhao S: MiR-145 sensitizes esophageal squamous cell carcinoma to cisplatin through directly inhibiting PI3K/AKT signaling pathway. Cancer Cell Int 19: 250, 2019.

16. Zhou J, Zheng S, Liu T, Liu Q, Chen Y, Tan D, Ma R and Lu X: $\mathrm{MCP} 2$ activates NF- $\kappa \mathrm{B}$ signaling pathway promoting the migration and invasion of ESCC cells. Cell Biol Int 42: 365-372, 2018.

17. Lu Z, Lu C, Li C, Jiao Y, Li Y and Zhang G: Dracorhodin perchlorate induces apoptosis and $\mathrm{G} 2 / \mathrm{M}$ cell cycle arrest in human esophageal squamous cell carcinoma through inhibition of the JAK2/STAT3 and AKT/FOXO3a pathways. Mol Med Rep 20: 2091-2100, 2019.

18. Wang WW, Zhao ZH, Wang L, Li P, Chen KS, Zhang JY, Li WC, Jiang GZ and Li XN: MicroRNA-134 prevents the progression of esophageal squamous cell carcinoma via the PLXNA1-mediated MAPK signalling pathway. EBioMedicine 46: 66-78, 2019.

19. Ferlay J, Soerjomataram I, Dikshit R, Eser S, Mathers C, Rebelo M, Parkin DM, Forman D and Bray F: Cancer incidence and mortality worldwide: Sources, methods and major patterns in GLOBOCAN 2012. Int J Cancer 136: E359-E386, 2015.

20. Wiedmann MW and Mössner J: New and emerging combination therapies for esophageal cancer. Cancer Manag Res 5: 133-146, 2013.

21. Ohashi S, Miyamoto S, Kikuchi O, Goto T, Amanuma Y and Muto M: Recent advances from basic and clinical studies of esophageal squamous cell carcinoma. Gastroenterology 149: 1700-1715, 2015 .
22. Hong H, Jie H, Liyu R, Zerui C, Borong S and Hongwei L: Prognostic significance of middle paraesophageal lymph node metastasis in resectable esophageal squamous cell carcinoma: A STROBE-compliant retrospective study. Medicine (Baltimore) 98 e17531, 2019.

23. Zhao Y, Han L, Zhang W, Shan L, Wang Y, Song P, Peng C and Zhao X: Preoperative chemotherapy compared to postoperative adjuvant chemotherapy for squamous cell carcinoma of the thoracic oesophagus with the detection of circulation tumour cells randomized controlled trial. Int J Surg 73: 1-8, 2020.

24. Zhang Z, Xu L, Di X, Zhang C, Ge X and Sun X: A retrospective study of postoperative radiotherapy for locally advanced esophageal squamous cell carcinoma. Ann Palliat Med 8: 708-716, 2019

25. Guan Q, Chen Z, Chen Q and Zhi X: XRCC1 and XPD polymorphisms and their relation to the clinical course in hepatocarcinoma patients. Oncol Lett 14: 2783-2788, 2017.

26. Yoon HH, Catalano PJ, Murphy KM, Skaar TC, Philips S, Powell M, Montgomery EA, Hafez MJ, Offer SM, Liu G, et al: Genetic variation in DNA-repair pathways and response to radiochemotherapy in esophageal adenocarcinoma: A retrospective cohort study of the eastern cooperative oncology group. BMC Cancer 11: 176, 2011.

27. Engin AB, Karahalil B, Engin A and Karakaya AE: DNA repair enzyme polymorphisms and oxidative stress in a Turkish population with gastric carcinoma. Mol Biol Rep 38: 5379-5386, 2011

28. Huang MY, Wang JY, Huang ML, Chang HJ and Lin SR Polymorphisms in XPD and ERCC1 associated with colorectal cancer outcome. Int J Mol Sci 14: 4121-4134, 2013.

29. Zheng JF, Li LL, Lu J, Yan K, Guo WH and Zhang JX: XPD functions as a tumor suppressor and dysregulates autophagy in cultures HepG2 cells. Med Sci Monit 21: 1562-1568, 2015.

30. Javadinia SA, Shahidsales S, Fanipakdel A, Mostafapour A, Joudi-Mashhad M, Ferns GA and Avan A: The esophageal cancer and the PI3K/AKT/mTOR signaling regulatory microRNAs: A novel marker for prognosis, and a possible target for immunotherapy. Curr Pharm Des 24: 4646-4651, 2018.

31. Han B, Jiang P, Li Z, Yu Y, Huang T, Ye X and Li X: Coptisine-induced apoptosis in human colon cancer cells (HCT-116) is mediated by PI3K/Akt and mitochondrial-associated apoptotic pathway. Phytomedicine 48: 152-160, 2018

32. Huang Y, Zhang J, Hou L, Wang G, Liu H, Zhang R, Chen X and Zhu J: LncRNA AK023391 promotes tumorigenesis and invasion of gastric cancer through activation of the PI3K/Akt signaling pathway. J Exp Clin Cancer Res 36: 194, 2017.

33. Parveen A, Akash MS, Rehman K and Kyunn WW: Dual role of p21 in the progression of cancer and its treatment. Crit Rev Eukaryot Gene Expr 26: 49-62, 2016.

34. Zhang D, Qi J, Liu R, Dai B, Ma W, Zhan Y and Zhang Y: c-Myc plays a key role in TADs-induced apoptosis and cell cycle arrest in human hepatocellular carcinoma cells. Am J Cancer Res 5: 1076-1088, 2015.

35. Cheng G, Zhang L, Lv W, Dong C, Wang Y and Zhang J: Overexpression of cyclin D1 in meningioma is associated with malignancy grade and causes abnormalities in apoptosis, invasion and cell cycle progression. Med Oncol 32: 439, 2015.

36. Alam M, Kashyap T, Pramanik KK, Singh AK, Nagini S and Mishra R: The elevated activation of NFKB and AP-1 is correlated with differential regulation of $\mathrm{Bcl}-2$ and associated with oral squamous cell carcinoma progression and resistance. Clin Oral Investig 21: 2721-2731, 2017.

37. Su F, Geng J, Li X, Qiao C, Luo L, Feng J, Dong X and Lv M: SP1 promotes tumor angiogenesis and invasion by activating VEGF expression in an acquired trastuzumab-resistant ovarian cancer model. Oncol Rep 38: 2677-2684, 2017.

38. Zhao G, Zhang H, Huang Z, Lv L and Yan F: Cortactin and Exo70 mediated invasion of hepatoma carcinoma cells by MMP-9 secretion. Mol Biol Rep 43: 407-414, 2016.

This work is licensed under a Creative Commons Attribution-NonCommercial-NoDerivatives 4.0 International (CC BY-NC-ND 4.0) License. 